This document is the Accepted Manuscript version of a Published Work that appeared in final form in Nano Letters, copyright (c) American Chemical Society after peer review and technical editing by the publisher. To access the final edited and published work see:

https://dx.doi.org/10.1021/acs. nanolett. 5b02635. 


\section{Manipulating the topological interface by molecular adsorbates: Adsorption of Co-Phthalocyanine on $\mathrm{Bi}_{2} \mathrm{Se}_{3}$}

Marco Caputo, ${ }^{* \dagger}{ }^{\dagger}$ Mirko Panighel, ${ }^{\ddagger}$ Simone Lisi, $₫$ Lama Khalil, ${ }^{\dagger}$ Giovanni Di

Santo,$^{\S}$ Evangelos Papalazarou, ${ }^{\dagger}$ Andrzej Hruban,, $\mid$ Marcin Konczykowski, ${ }^{\perp}$ Lia

Krusin-Elbaum,\# Pranab Kumar Das, ${ }^{@}$ Jun Fuji, ${ }^{@}$ Ivana Vobornik, ${ }^{@}$ Luca

Perfetti, $\triangle$ Aitor Mugarza, ${ }^{\ddagger}$ Andrea Goldoni, ${ }^{\S}$ and Marino Marsi ${ }^{\dagger}$

$\dagger$ †aboratoire de Physique des Solides, CNRS-UMR 8502, Universitè Paris-Sud, 91405

Orsay, France

$\ddagger$ Catalan Institute of Nanoscience and Nanotechnology (ICN2), CSIC and The Barcelona

Institute of Science and Technology, Campus UAB, Bellaterra, 08193 Barcelona, Spain

ICREA - Instituciò Catalana de Recerca i Estudis Avancats, Lluis Companys 23, 08010

Barcelona, Spain

IDipartimento di Fisica, Università di Roma La Sapienza, Piazzale A. Moro 5, 00185

Roma, Italy

$\S$ Laboratory Micro $\& 3$ Nano Carbon Consorzio INSTM UdR Trieste-ST c/o ELETTRA -

Sincrotrone Trieste S.C.p.A. s.s.14, Km 163.5, 34149 Trieste, Italy

|| Institute of Electronic Materials Technology, 01-919 Warsaw, Poland

$\perp$ Laboratoire des Solides Irradiés, CNRS-UMR 7642 CEA-DSM-IRAMIS, Ecole

Polytechnique, 91128 Palaiseau, France

\#Department of Physics, The City College of New York, CUNY, New York, NY 10031,

$U S A$

@Istituto Officina dei Materiali (IOM)-CNR, Laboratorio TASC, Area Science Park, s.s.14, Km 163.5, 34149 Trieste, Italy

$\triangle$ Laboratoire des Solides Irradiés, Ecole polytechnique, 91128 Palaiseau, France

E-mail: marco.caputo@u-psud.fr 


\begin{abstract}
Topological insulators are a promising class of materials for application in the field of spintronics. New perspectives in this field can arise from interfacing metalorganic molecules with the topological insulators spin-momentum locked surface states, which can be perturbed enhancing or suppressing spintronics-relevant properties such as spin coherence. Here we show results from an Angle Resolved Photemission Spectroscopy (ARPES) and Scanning Tunnelling Microscopy (STM) study of the prototypical Cobalt Phthalocyanine $(\mathrm{CoPc}) / \mathrm{Bi}_{2} \mathrm{Se}_{3}$ interfaces. We demonstrate that that the hybrid interface can act on the topological protection of the surface and bury the Dirac Cone below the first quintuple layer.
\end{abstract}

\title{
Keywords
}

Topological insulator, Phthalocyanine, ARPES, Surface states, Dirac cone, Charge transfer 
In these last years topological insulators received the attention of a large area of the scientific community thanks to their exotic properties. Topological insulators are semiconductors in the bulk, but they posses metallic surface states topologically protected by time-reversal symmetry. Moreover, these surface states are spin-momentum locked, meaning that for instance no backscattering is allowed for electrons occupying these states. ${ }^{1-3}$ Thanks to these properties topological insulators are promising materials for the field of spintronics, where they can be exploited to implement spin generator, transport, or torque devices. ${ }^{4-8}$

Metalorganic molecules are another very promising material for spintronic applications. It has already been demonstrated that metalorganic molecules can be used as spin valves, molecular switches, and other devices. ${ }^{9-11}$ Among all metalorganic complexes, phorphyirines and phthalocyanines $(\mathrm{Pc})$ are the most studied systems. These are simple molecules, composed of a macrocycle that can host a central metal atom. Replacing the central atom will result in changes in the molecular electronic structure, and in the case of half-filled d-orbital metals (i.e. Mn, Fe, and Co) interesting magnetic properties arise. ${ }^{12-15}$

A new class of spintronic devices could derive by the interplay between topological insulators and metalorganic molecules, and in this sense fundamental studies of this interface are highly desirable. Scanning tunnelling microscopy-spectroscopy (STM-STS) studies of manganese phthalocyanine $(\mathrm{MnPc})$, cobalt phthalocyanine $(\mathrm{CoPc})$, and copper phthalocyanine $(\mathrm{CuPc}) / \mathrm{Bi}_{2} \mathrm{Te}_{3}$ show that a metal center-dependent interaction between molecule and substrate exists, ${ }^{16,17}$ as already reported for transition metal phthalocyanine (TMPc)/Au(110) and $(\mathrm{TMPc}) / \operatorname{Ag}(100) .{ }^{18,19}$ An evidence of hybridization between unoccupied molecular orbitals and substrate states has been reported for $\mathrm{MnPc} / \mathrm{Bi}_{2} \mathrm{Te}_{3}$, nevertheless in this case the substrate surface states are unaffected by the molecular adsorption. ${ }^{16} \mathrm{~A}$ similar study has been also carried out by Song at al., ${ }^{20}$ where one monolayer (1 ML) of iron phthalocyanine (FePc) has been deposited on $\mathrm{Bi}_{2} \mathrm{Te}_{3}$ surface. An in-plane easy magnetization axis for $\mathrm{FePc}$ has been observed, however no signs of spontaneous magnetic ordering has been found for this system. 
These erlier experimental studies were focused on the molecular point of view, without the direct access to the effects of molecular adsorption on the topological insulator surface band structure that can be given by an angle resolved photoemission (ARPES) study. In a recent work Jakobs et al. dealt with this issue with a mixed theoretical and experimental aproach: ${ }^{21}$ they showed that depending on the molecule-surface interaction strength Rashbasplit interface states can arise. Up to now, however, no such studies have been performed on metalorganic complexes. Adding metal atoms to the organic framework can introduce new effects: magnetic impurities and metalorganic complexes on the surface of a topological insulator are predicted to break the time reversal protection of the surface state, ${ }^{22-25}$ however previous investigations of the effect of metal adsorption on the surface states of $\mathrm{Bi}_{2} \mathrm{Se}_{3}$ showed the opposite. Wray et al. claimed to have observed the breaking of time reversal symmetry and the consequent opening of a gap in the surface states upon Fe deposition, ${ }^{26}$ but this interpretation was successively questioned explaining the observation by a simple band bending effect and simultaneous confinement of both valence and conduction bands. ${ }^{27}$ Indeed, other experiments performed in similar conditions did not confirm any gap opening. ${ }^{28-31}$

In order to shed light on the effects of the adsorbtion of metalorganic molecules on the surface of a topological insulator, we performed a combined a angle resolved photoemission spectroscopy and scanning tunnelling microscopy investigation of cobalt phthalocyanine adsorbed on the prototypical topological insulator $\mathrm{Bi}_{2} \mathrm{Se}_{3}$. We chose $\mathrm{Bi}_{2} \mathrm{Se}_{3}$ because its Dirac point is located in the bandgap, and this is a prerequisite for the realization of the anomalous quantum hall effect and other magnetoelectric phenomena. Futhermore, this surface is better suited for ARPES experiments on the Dirac point than $\mathrm{Bi}_{2} \mathrm{Te}_{3}$ since it allows its direct visualization away from the band bulk. The interface electronic structure upon CoPc adsorption was studied using ARPES with different photon energy, and so probing depths. Our results indicate that the topological surface stage (TSS) survives in the subsurface region, while it is disrupted in the first quintuple layer.

Figure 1 shows an overview of the evolution of the electronic structure of the interface 
upon $\mathrm{CoPc}$ adsorption acquired with a photon energy of $55 \mathrm{eV}$. Increasing the coverage
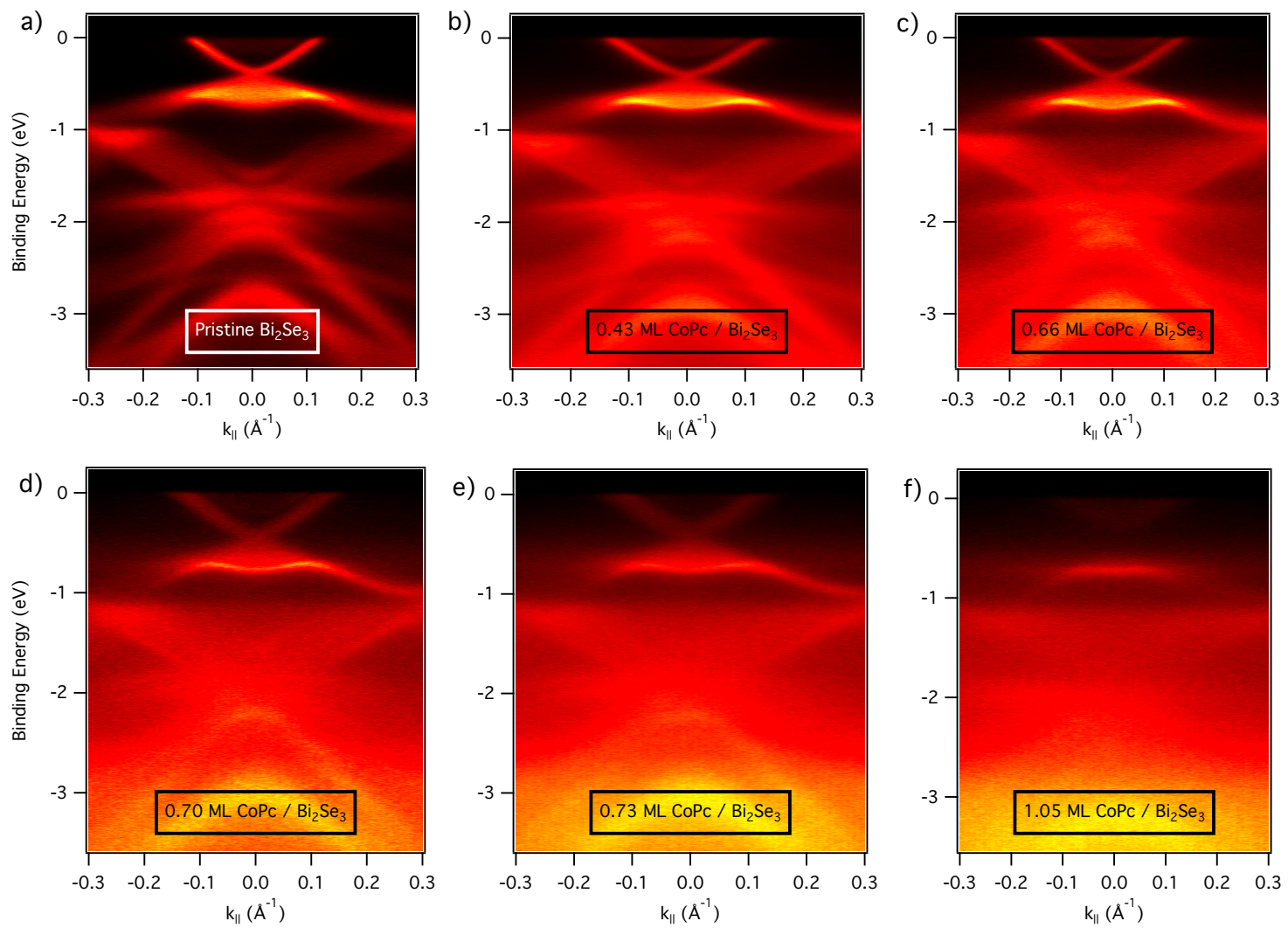

Figure 1: ARPES spectra for $\mathrm{CoPc} / \mathrm{Bi}_{2} \mathrm{Se}_{3}$ at $h \nu=55 \mathrm{eV}$. Intensity is coded in colours, from black (lowest) to red and to yellow (highest). CoPc coverage is indicated in each panel.

(from panel a) to panel $\mathrm{f}$ )) results initially in a blurring of the $\mathrm{Bi}_{2} \mathrm{Se}_{3}$ bulk states that could be related to hybridization with molecular orbitals and/or scattering from the randomly distributed molecules on the surface. At higher coverage some almost dispersionless features appear at approximately $0.72 \mathrm{eV}, 1.22 \mathrm{eV}, 1.98 \mathrm{eV}, 3.17 \mathrm{eV}$ binding energy. These features can be assigned to the $\mathrm{CoPc} / \mathrm{Bi}_{2} \mathrm{Se}_{3}$ interface states: previous investigation on a monolayer of $\mathrm{CoPc} / \mathrm{Au}(110)$ and $\mathrm{CoPc} / \mathrm{Au}$-poly reported analogous binding energy for these features. ${ }^{32,33}$

The case of one monolayer of $\mathrm{CoPc} / \mathrm{Cu}(111)$ is different. ${ }^{34}$ This interface shows the same emerging features, but they are shifted of about $0.15 \mathrm{eV}$ toward higher binding energy. In this case a charge transfer from the substrate to the molecules has been easily observed as a shift towards lower binding energy of the $\mathrm{Cu}(111)$ surface Shockley state. In the case of 
$\mathrm{CoPc} / \mathrm{Bi}_{2} \mathrm{Se}_{3}$ we observed exactly the opposite occurrence: already from the lowest coverage a clear downshift of the Dirac cone due to a charge transfer from the molecule to the surface is evident. Figure $2 \mathrm{a}-\mathrm{b}$ show the comparison between the clean surface and $0.66 \mathrm{ML}$ $\mathrm{CoPc} / \mathrm{Bi}_{2} \mathrm{Se}_{3}$ : a clear downshift of $50 \mathrm{meV}$ of the surface state is visible. Our measurements
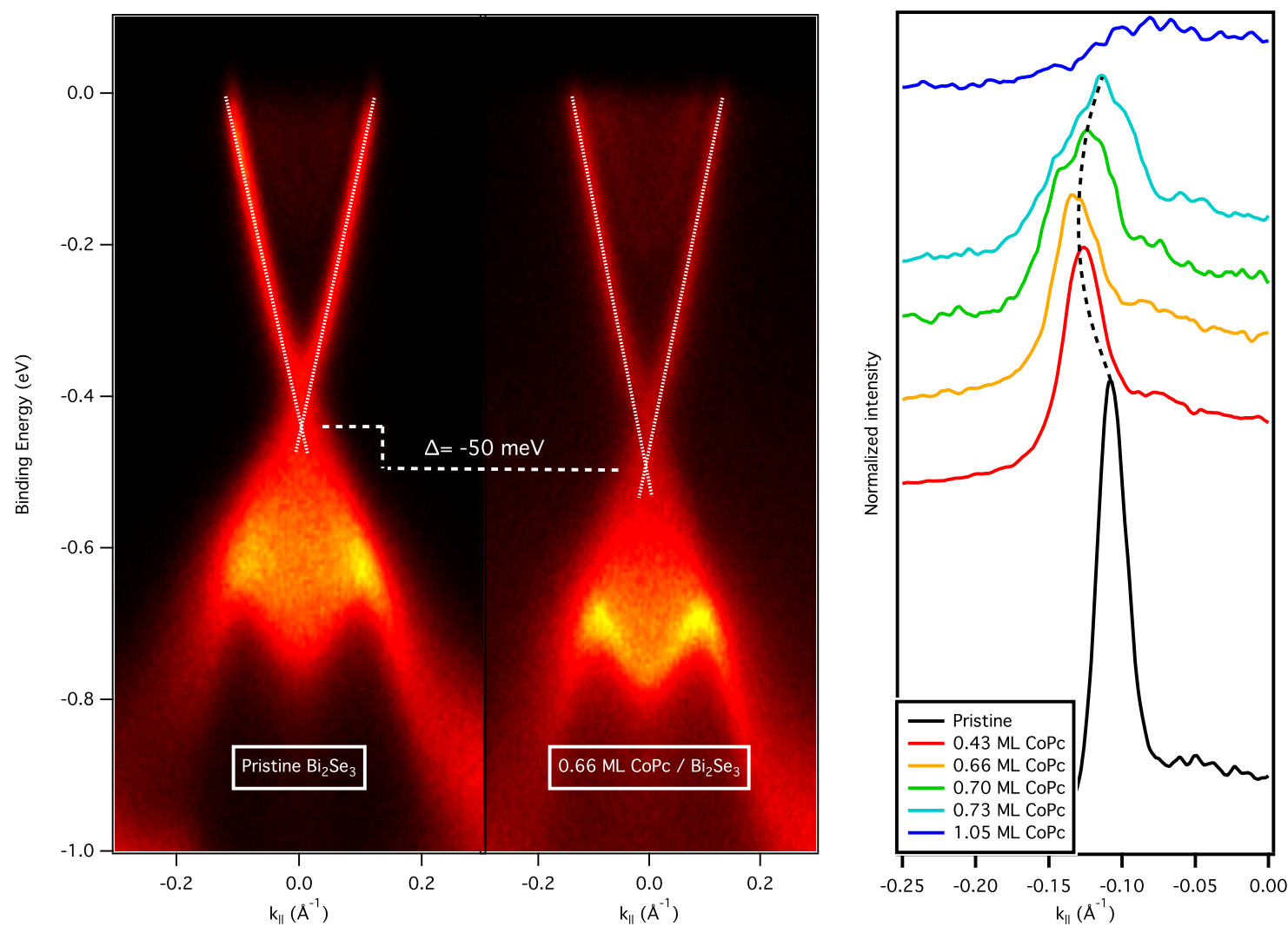

Figure 2: a)-b) Enlargement near the Dirac point for the pristine surface and for 0.66 ML $\mathrm{CoPc} / \mathrm{Bi}_{2} \mathrm{Se}_{3}$. c) MDC curves taken integrating the region $0-0.03 \mathrm{eV}$ of binding energy and normalized to the bulk pocket intensity. Dashed lines are guides for eyes.

show that when the coverage exceeds the value of 0.66 the charge transfer decreases. At higher coverage the increasingly blurry features prevent a reliable estimation of the actual Dirac point position, however we can recall a qualitative trend from the momentum distribution curves (MDC) acquired at the Fermi level shown in panel c of figure 2: Fermi momentum increases up to the coverage of $0.66 \mathrm{ML}$, when it reaches its maximum, while with further deposition it decreases. The Fermi momentum is clearly related to the energy 
position of the Dirac point: a downshift of the latter causes the Dirac cone to be cut by the Fermi level further with respect to its vertex, where distance between the two branches, and thus the Fermi momentum, is higher. On the contrary, we can clearly see that for coverages higher than 0.66 ML the Fermi momentum moves toward lower values: this is an indication that the Substrate energy levels are shifting now to lower binding energy. This could be an indication of a coverage dependent interaction between surface and molecules. Different studies on MPc on $\mathrm{Au}$ and $\mathrm{Ag}$ showed that molecule-substrate distance changes depending on the molecular coverage, increasing for coverages approaching 1ML. ${ }^{35-37}$ An increase in the molecule-substrate distance turns in a decoupling between the two that in this case can be observed as a reduction of the charge transfer. Futhuremore, this behavior allows us to rule out spurious effects related to surface contamination: in this case the electron doping varies continuously up to a saturation value, where it remains. ${ }^{38}$

Once the monolayer has been completed the topologically protected surface states seems to vanish from the surface sensitive ARPES signal. Figure 3 shows a detail of the region 0-1 eV of binding energy for the last two depositions: $0.73 \mathrm{ML} \mathrm{CoPc} / \mathrm{Bi}_{2} \mathrm{Se}_{3}$ and $1 \mathrm{ML}$ $\mathrm{CoPc} / \mathrm{Bi}_{2} \mathrm{Se}_{3}$. In panel a) (corresponding to $0.73 \mathrm{ML} \mathrm{CoPc} / \mathrm{Bi}_{2} \mathrm{Se}_{3}$ ) surface states, although blurry, are still clearly discernible, along with the bulk valence and conduction band. Adding more molecules, up to complete one monolayer, results in an abrupt change in the band structure. Panel b of Figure 3 shows that, while bulk conduction and valence bands of $\mathrm{Bi}_{2} \mathrm{Se}_{3}$ are still visible, the Dirac cone between the two disappeared. Of course the presence of both $\mathrm{Bi}_{2} \mathrm{Se}_{3}$ bulk valence and conduction bands ensures that the electron inelastic mean free path is sufficient to make outgoing electrons overcome the molecular layer and reach the vacuum.

It is worth noting that also the shape of the bulk valence band seems to undergo a drastic change: this is due to the superposition of the non-dispersing interface state at $0.72 \mathrm{eV}$ with the bulk valence band, which hinders the dispersion of the latter.

Of course the disappearance of the ARPES signal coming from the topologically protected 

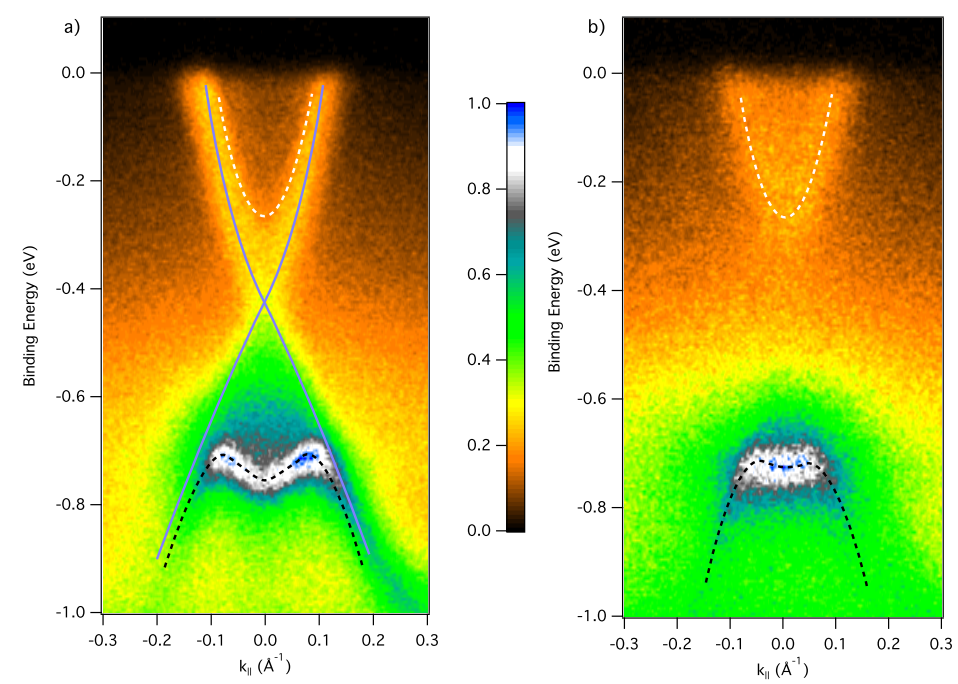

Figure 3: Detail of the region 0-1 eV of binding energy for $0.73 \mathrm{ML} \mathrm{CoPc} / \mathrm{Bi}_{2} \mathrm{Se}_{3}$ (panel $\mathrm{a}$, left) and $1 \mathrm{ML} \mathrm{CoPc} / \mathrm{Bi}_{2} \mathrm{Se}_{3}$ (panel b, right). Lines are guide for eyes indicating $\mathrm{Bi}_{2} \mathrm{Se}_{3}$ surface states (blue continuous line), $\mathrm{Bi}_{2} \mathrm{Se}_{3}$ bulk valence band (black dashed line), and $\mathrm{Bi}_{2} \mathrm{Se}_{3}$ bulk conduction band (white dashed line).

surface states does not imply that these do not exist anymore. One of the options to be considered is that their wavefunction is perturbed by the hybridization between molecular orbitals and surface states so that they disappear form the first quintuple layer. However, the surface states in 3D topological insulators is known to penetrate in the bulk for at least 2 quintuple layers, ${ }^{39,40}$ so, due to the robust topological protection, the tail of the surface states extending into the second quintuple layer can remain unperturbed. The images shown up to now were acquired with a photon energy of $55 \mathrm{eV}$, where the electron inelastic mean free path (IMFP) can be estimated between 3 and $6 \AA,{ }^{41}$ less than the height of one quintuple layer (approximatively $1 \mathrm{~nm}^{42}$ ). By changing the photon energy it is possible to increase the probing depth of our experiment. Figure 4 shows a comparison of a pristine $\mathrm{Bi}_{2} \mathrm{Se}_{3}$ surface (panel a) and a $\mathrm{CoPc} / \mathrm{Bi}_{2} \mathrm{Se}_{3}$ interface (panel b) acquired with a photon energy of $6.28 \mathrm{eV}$. At this energy the estimated IMFP approaches $20 \AA,{ }^{43}$ enough to access the first and the second quintuple layer, and Figure 4 shows clearly that in this condition the Dirac cone is still clearly discernible. Well-resolved surface states enable us also to estimate in 10 


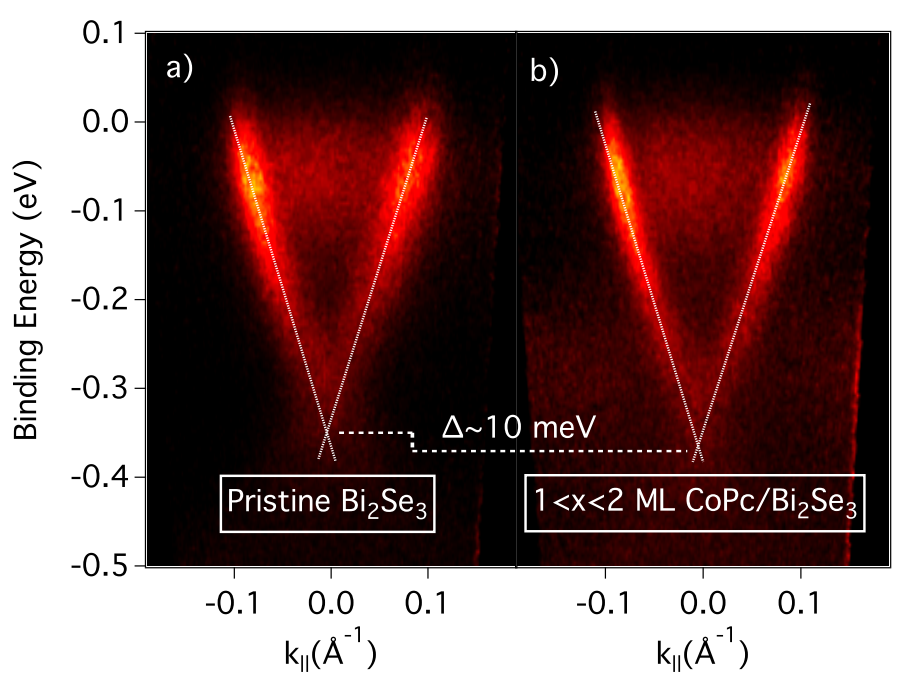

Figure 4: Comparison between a pristine $\mathrm{Bi}_{2} \mathrm{Se}_{3}$ surface (panel a) and a $\mathrm{CoPc} / \mathrm{Bi}_{2} \mathrm{Se}_{3}$ interface (panel b) acquired with a photon energy of $6.28 \mathrm{eV}$. Overlayer thickness is estimated to be in the range between one and two ML. White dashed lines are guide for eyes

meV their energy shift once a complete monolayer of $\mathrm{CoPc}$ is formed, much less than the 50 meV observed in the case of $0.66 \mathrm{ML}$. This is consistent with the qualitative trend showed in Figure 2 and also with the data presented by Bathon et al., ${ }^{17}$ where a negligible charge transfer is reported.

The effect of CoPc adsorption is dramatically different with respect to the effect of metalfree phthalocyanine $^{21}$ (see also supporting information) and of metal atoms deposition. Other attempts under similar conditions, with coverages ranging from a few per cent to some tenths of a monolayer of iron ${ }^{29,31}$ and cobalt $^{28}$ did not lead to anything similar to what is shown here: surface metallic doping seems to have no effects on the topologically protected states. However, for a direct comparison between our experiment and previous surface doping studies, the equivalent coverage of Co atoms has to be known. STM topogrphy in Figure 5 show that the molecules are arranged in a self-assembled monolayer with a similar hexagonal herringbone-distorted geometry previously observed for $\mathrm{CoPc} / \mathrm{Bi}_{2} \mathrm{Te}_{3}{ }^{17}$. The intermetallic distance in this case is $1.28 \mathrm{~nm}$, and assuming $0.25 \mathrm{~nm}$ as lattice constant for a Co film grown on the $\mathrm{Bi}_{2} \mathrm{Se}_{3}$ surface (the same lattice constant of bulk Co crystal with also the 


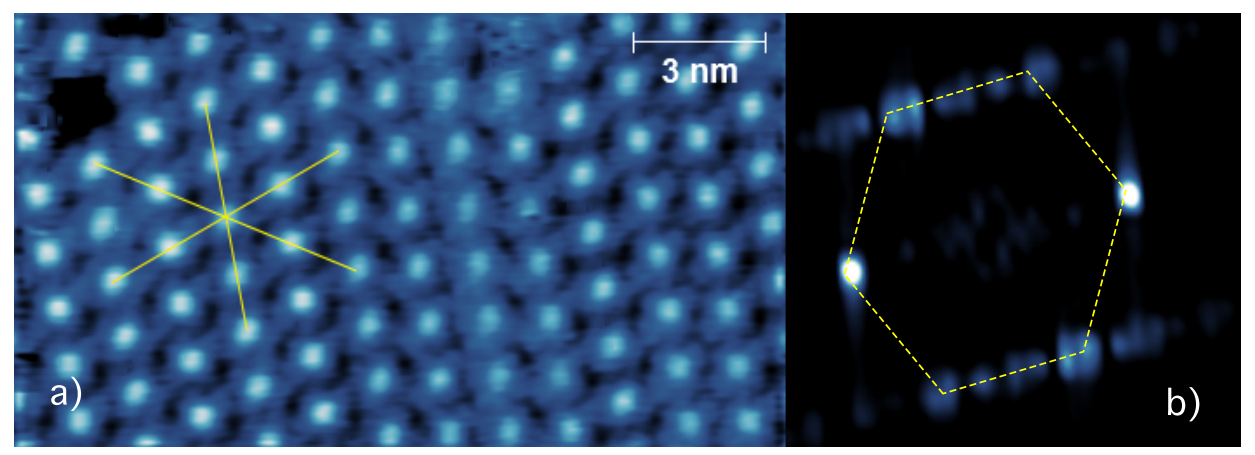

Figure 5: a) STM image of a monolayer of CoPc acquired at a $\mathrm{V}_{\text {bias }}=2.78 \mathrm{~V}$ e $\mathrm{I}_{\mathrm{t}}=14 \mathrm{pA}$. b) Fourier transform of the panel a). Yellow lines indicate the overlayer symmetry.

same hexagonal symmetry), a nominal coverage of $0.04 \mathrm{ML}$ of cobalt atoms is obtained, comparable to the previous surface doping experiments.

The different behaviour observed for $\mathrm{CoPc} / \mathrm{Bi}_{2} \mathrm{Se}_{3}$ in contrast with all other surface-doped systems underlines once again the flexibility inherent of metalorganic networks. These are capable of avoiding clustering of metal atoms, induce crystal field splitting, and in general modify the metal-surface interaction. All these factors may lead to very different interfacial interactions and corresponding perturbation of the TSS as compared to metallic impurity doped surfaces, as observed in the present work.

In conclusion, we studied the electronic structure modification induced by the adsorption of different coverage of cobalt phthalocyanine on $\mathrm{Bi}_{2} \mathrm{Se}_{3}$ surface. First ARPES results point towards the existence of a relevant interaction between the molecule and the surface underneath, with the effect of burying the wave function of the TSS below the first quintuple layer. Future studies are required to understand the mechanism of this occurrence. One possibility is that the metalorganic network is capable of triggering a surface-mediated interaction between the metal centres of the molecules as proposed by some theoetical works, ${ }^{22-24}$ which could be elucidated by x-ray magnetic circular dichroism experiments. Our results show the potential of metalorganic molecules for the control of Dirac fermions in topologically protected states through the metal-metal as well as the metal-substrate interaction, which could be further tuned by adding different functional groups to the ligand. ${ }^{44}$ 


\section{Acknowledgement}

The authors thank A. Politano, Z. S. Aliev, M. B. Babanly, A. Arnau, and E. V. Chulkov for providing a first batch of $\mathrm{Bi}_{2} \mathrm{Se}_{3}$ samples used to start the experiment.

Authors warmly thank also P. Sessi and M. Cinchetti for fruitful discussion.

\section{Supporting Information Available}

Experimental method, XPS deposition calibration, ARPES data on freshly prepared 1ML $\mathrm{CoPc} / \mathrm{Bi}_{2} \mathrm{Se}_{3}$ sample, and an anolgous experiment on $2 \mathrm{H}-\mathrm{Pc}$.

This material is available free of charge via the Internet at http://pubs.acs.org/.

\section{References}

(1) Hasan, M. Z.; Kane, C. L. Rev. Mod. Phys. 2010, 82, 3045-3067.

(2) Zhang, H.; Liu, C.-X.; Qi, X.-L.; Dai, X.; Fang, Z.; Zhang, S.-C. Nat. Phys. 2009, 5, 438-442.

(3) Hsieh, D. et al. Nature 2009, 460, 1101-1105.

(4) Vobornik, I.; Manju, U.; Fujii, J.; Borgatti, F.; Torelli, P.; Krizmancic, D.; Hor, Y. S.; Cava, R. J.; Panaccione, G. Nano Lett. 2011, 11, 4079-4082.

(5) Burkov, a. a.; Hawthorn, D. G. Phys. Rev. Lett. 2010, 105, 6-9.

(6) Mellnik, a. R.; Lee, J. S.; Richardella, a.; Grab, J. L.; Mintun, P. J.; Fischer, M. H.; Vaezi, a.; Manchon, a.; Kim, E.-a.; Samarth, N.; Ralph, D. C. Nature 2014, 511, 449-451.

(7) Fan, Y. et al. Nat. Mater. 2014, 13, 699-704.

(8) Pesin, D.; MacDonald, A. H. Nat. Mater. 2012, 11, 409-416. 
(9) Xiong, Z. H.; Wu, D.; Vardeny, Z. V.; Shi, J. Nature 2004, 427, 821-824.

(10) Auwärter, W.; Seufert, K.; Bischoff, F.; Ecija, D.; Vijayaraghavan, S.; Joshi, S.; Klappenberger, F.; Samudrala, N.; Barth, J. V. Nat. Nanotechnol. 2012, 7, 41-6.

(11) Rocha, A. R.; García-Suárez, V. M.; Bailey, S. W.; Lambert, C. J.; Ferrer, J.; Sanvito, S. Nat. Mater. 2005, 4, 335-339.

(12) Wende, H. et al. Nat. Mater. 2007, 6, 516-520.

(13) Zhou, J.; Sun, Q. J. Am. Chem. Soc. 2011, 133, 15113-15119.

(14) Krull, C.; Robles, R.; Mugarza, A.; Gambardella, P. Nat. Mater. 2013, 12, 337-343.

(15) Lisi, S.; Gargiani, P.; Scardamaglia, M.; Brookes, N. B.; Sessi, V.; Mariani, C.; Betti, M. G. J. Phys. Chem. Lett. 2015, 6, 1690-1695.

(16) Sessi, P.; Bathon, T.; Kokh, K.; Tereshencko, O.; Bode, M. Nano Lett. 2014, 14, 50925096.

(17) Bathon, T.; Sessi, P.; Kokh, K. a.; Tereshchenko, O. E.; Bode, M. Nano Lett. 2015, 15, 2442-2447.

(18) Mugarza, a.; Robles, R.; Krull, C.; Korytár, R.; Lorente, N.; Gambardella, P. Phys. Rev. B 2012, 85, 1-13.

(19) Massimi, L.; Angelucci, M.; Gargiani, P.; Betti, M. G.; Montoro, S.; Mariani, C. J. Chem. Phys. 2014, 140, 244704.

(20) Song, Y.; Zhang, Y.; Yang, F.; Zhang, K.; Liu, C. Phys. Rev. B. 2014, 90, 180408.

(21) Jakobs, S. et al. Nano Lett. 2015, 15, 6022-6029.

(22) Liu, Q.; Liu, C.-X.; Xu, C.; Qi, X.-L.; Zhang, S.-C. Phys. Rev. Lett. 2009, 102, 156603.

(23) Biswas, R. R.; Balatsky, a. V. Phys. Rev. B. 2010, 81, 233405. 
(24) Abanin, D. a.; Pesin, D. a. Phys. Rev. Lett. 2011, 106, 136802.

(25) Otrokov, M. M.; Chulkov, E. V.; Arnau, A. Phys. Rev. B 2015, 92, 165309.

(26) Wray, L. A.; Xu, S.-Y.; Xia, Y.; Hsieh, D.; Fedorov, A. V.; Hor, Y. S.; Cava, R. J.; Bansil, A.; Lin, H.; Hasan, M. Z. Nat. Phys. 2010, 7, 32-37.

(27) Bianchi, M.; Hatch, R. C.; Mi, J.; Iversen, B. B.; Hofmann, P. Phys. Rev. Lett. 2011, $107,2-5$.

(28) Ye, M. et al. Phys. Rev. B. 2012, 85, 205317.

(29) Scholz, M. R.; Sánchez-Barriga, J.; Marchenko, D.; Varykhalov, A.; Volykhov, A.; Yashina, L. V.; Rader, O. Phys. Rev. Lett. 2012, 108, 256810.

(30) Honolka, J.; Khajetoorians, a. a.; Sessi, V.; Wehling, T. O.; Stepanow, S.; Mi, J. L.; Iversen, B. B.; Schlenk, T.; Wiebe, J.; Brookes, N. B.; Lichtenstein, a. I.; Hofmann, P.; Kern, K.; Wiesendanger, R. Phys. Rev. Lett. 2012, 108, 256811.

(31) Schlenk, T.; Bianchi, M.; Koleini, M.; Eich, A. Phys. Rev. Lett. 2013, 110, 126804.

(32) Gargiani, P.; Angelucci, M.; Mariani, C.; Betti, M. G. Phys. Rev. B 2010, 81, 085412.

(33) Petraki, F.; Peisert, H.; Biswas, I.; Chasse, T. J. Phys. Chem. C 2010, 114, $17638-$ 17643.

(34) Annese, E.; Fujii, J.; Vobornik, I.; Rossi, G. J. Phys. Chem. C 2011, 115, 17409-17416.

(35) Cheng, Z. H.; Gao, L.; Deng, Z. T.; Jiang, N.; Liu, Q.; Shi, D. X.; Du, S. X.; Guo, H. M.; Gao, H. J. J. Phys. Chem. C 2007, 111, 9240-9244.

(36) Gopakumar, T. G.; Brumme, T.; Kröger, J.; Toher, C.; Cuniberti, G.; Berndt, R. J. Phys. Chem. C 2011, 115, 12173-12179. 
(37) Kroeger, I.; Stadtmueller, B.; Stadler, C.; Ziroff, J.; Kochler, M.; Stahl, A.; Pollinger, F.; Lee, T.; Zegenhagen, J.; Reinert, F.; Kumpf, C. New J. Phys. 2010, 12, 83038.

(38) Bianchi, M.; Hatch, R. C.; Guan, D.; Planke, T.; Mi, J.; Iversen, B. B.; Hofmann, P. Semicond. Sci. Technol. 2012, 27, 124001.

(39) Zhang, Y. et al. Nat. Phys. 2010, 6, 584-588.

(40) Dai, X. Q.; Zhao, B.; Zhao, J. H.; Li, Y. H.; Tang, Y. N.; Li, N. J. Phys.: Condens. Matter 2012, 24, 35502.

(41) Seah, M. P.; Dench, W. A. Surf. Interface Anal. 1979, 1, 2-11.

(42) Mishara, S. K.; Satpathy, S.; Jepsen, O. J. Phys.: Condens. Matter 1997, 9, 461.

(43) Offi, F.; Iacobucci, S.; Vilmercati, P.; Rizzo, A.; Goldoni, A.; Sacchi, M.; Panaccione, G. Phys. Rev. B 2008, 77, 5-8.

(44) Gottfried, M.; Marbach, H. Phys. Chem.: Int. J. Res. Phys. Chem. Chem. Phys. 2009, 223, 53-74. 
Graphical TOC Entry

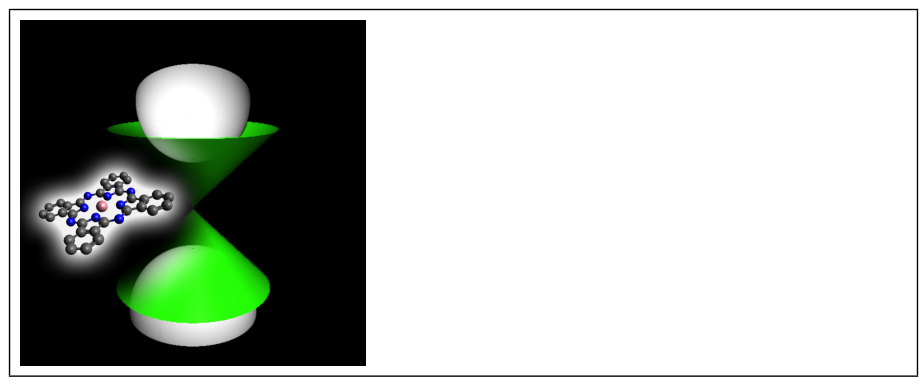

